\title{
INTERSTELLAR DUST IN COLLECTED INTERPLANETARY DUST PARTICLES
}

\author{
SCOTT A. SANDFORD \\ NASA/Ames Research Center, Moffett Field, CA 94095
}

\begin{abstract}
During the past decade interplanetary dust particles (IDPs) have been collected in the earth's stratosphere. Isotopic studies of these particles have demonstrated that many of them are greatly enriched in deuterium and at least some of them carry this enrichment in smaller subcomponents. Deuterium enrichments of a similar magnitude are seen in simple molecules in interstellar clouds. Deuterium enrichment in IDPs can be taken as evidence for the presence of interstellar material. It is not clear at this time whether the carriers of the isotopic anomalies represent true, unaltered interstellar dust grains, or whether they represent an altered component with a molecular 'memory' of original interstellar grains. The spectra of different components in the collected dust provide suggestive matches to similar components evident in the astronomical spectra of dust in comets, dense molecular clouds, and emission nebulae. The known extraterrestrial nature of the particles, the possible presence of interstellar material in them, and their spectral similarity to many astronomical objects all argue that the collected IDPs provide useful analogs for the modelling of interstellar dust.
\end{abstract}

\section{INTRODUCTION}

In the past decade it has become possible to collect relatively pristine samples of interplanetary dust from the earth's stratosphere using high altitude aircraft. The collected interplanetary dust particles (IDPs) have diameters in the 5 to $50 \mu \mathrm{m}$ range and they are deaccelerated high in the earth's atmosphere without suffering catastrophic melting or vaporization. Once decelerated, these particles slowly settle out of the atmosphere and can be collected in the stratosphere before they become fully mixed with the terrestrial particulate material commonly found at lower altitudes. As part of a program originally started by Dr. Donald Brownlee of Washington University in Seattle, these particles are collected on impact collectors mounted on the wings of high altitude NASA aircraft. Exposed collectors can then be examined in a cleanroom and the particles removed for further investigation. It is now well established that several subsets of the dust found on these collectors are extraterrestrial. The most common type of IDP found on the collectors is referred to as "chondritic" because the observed relative abundances of the major elements in these IDPs are similar to those of primitive meteorites called carbonaceous chondrites (i.e. they have roughly solar abundance values). There are several independent proofs of the extraterrestrial nature of the chondritic particles. First, the crystalline minerals in many of these particles contain heavy-element ion tracks, demonstrating that 


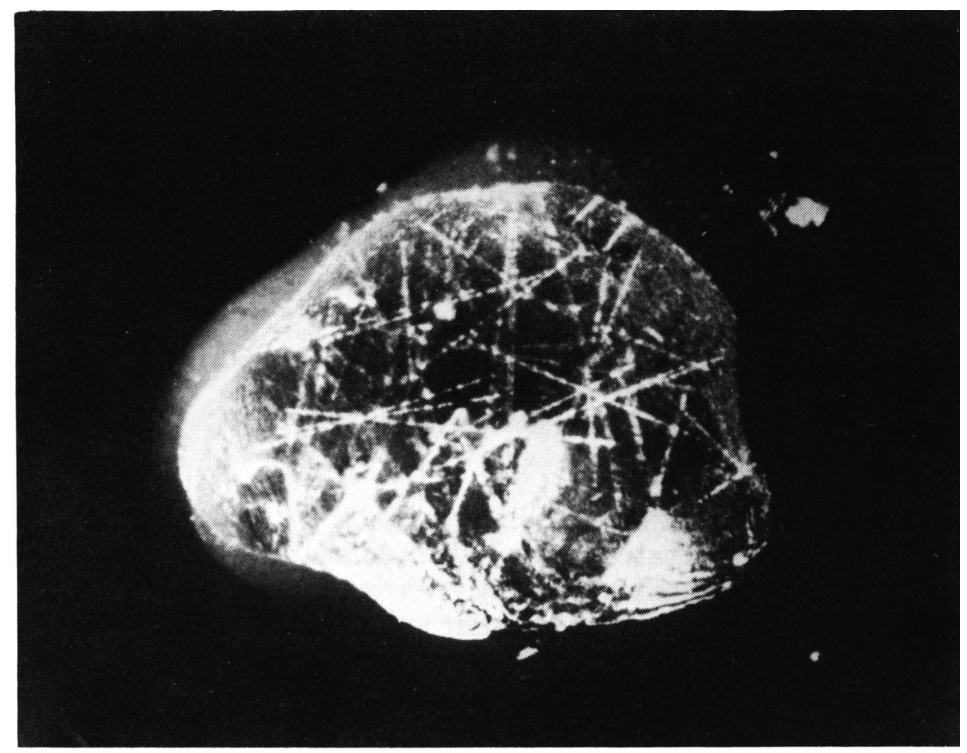

Fig. 1. Transmission electron microscope photo of a single mineral grain within an IDP. The randomly oriented tracks within the grain were produced by solar flare ions. The grain is approximately 0.5 microns across. (Photo courtesy of J. Bradley, McCrone Associates).

they have been exposed in space to solar flare particles. Some of the particles also show thin amorphous rims along their outer edges that are thought to be due to solar wind irradiation (Figure 1). In addition, the noble gases in the particles have been found to have abundances different from the terrestrial atmosphere. Finally, many of the IDPs have been shown to contain non-terrestrial isotopic anomalies in the elements hydrogen and oxygen. For more complete discussions of the collection and curation of the stratospheric IDPs and proof of their extraterrestrial origins, the reader is referred to reviews by Fraundorf, Brownlee, and Walker (1982), Sandford (1987), Mackinnon and Rietmeijer (1987), and Bradley, Sandford, and Walker (1988).

\section{IDP MINERALOGY AND MORPHOLOGY}

Elemental, mineralogical, and spectral studies have all demonstrated that the collected chondritic IDPs can be classified into several distinct groups. The first major group of chondritic IDPs, and the most abundant in the collections, contains particles dominated by hydrated layer-lattice silicates. The most commonly found layer-lattice silicates are smectites, although a few particles have been found which contain mostly serpentine. This is the opposite of what is seen in most primitive meteorites where serpentine is the dominant layer-lattice silicate. As can be seen in Figure 2a, which shows an IDP of this type, these particles are irregular in shape and tend to be relatively compact. They contain little internal void space. Particles 

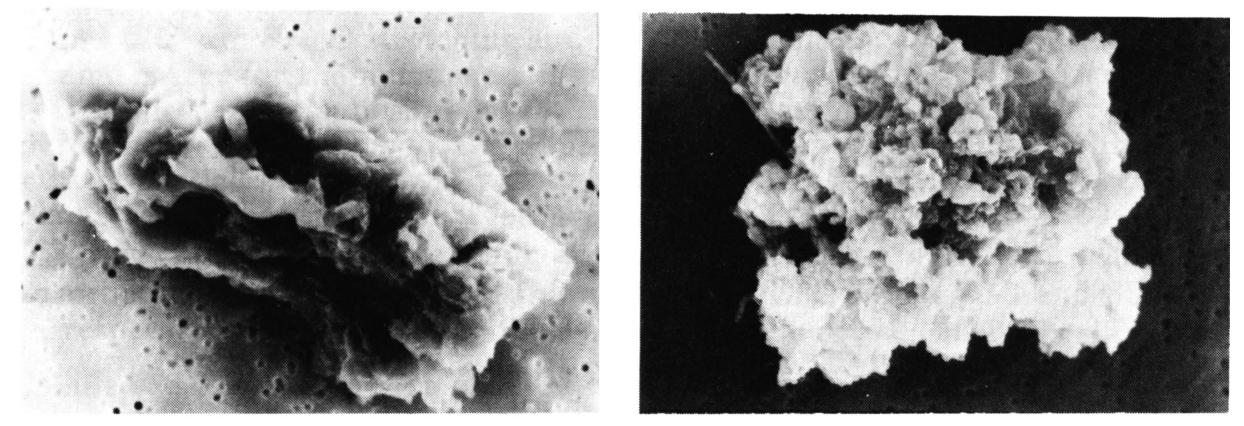

Fig. 2. (a) Scanning electron microscope photo of a typical IDP in the layer-lattice silicate class. These particles tend to be compact. The scale bar is 1 micron long. (b) Scanning electron microscope photo of a typical IDP in the class containing the anhydrous minerals olivine and pyroxene. These particles appear to be the product of the aggregation of precursor grains and are typically porous. The scale bar is 10 microns long. (Photos courtesy of J. Bradley, McCrone Associates).

falling in the second chondritic IDP group are dominated by the anhydrous minerals olivine and pyroxene. These particles tend to show a morphological structure that suggests the random aggregation of smaller grains (Figure 2b). Examination of thin sections of these particles shows that these IDPs contain a wide variety of crystalline mineral grains intermixed within a 'matrix' of amorphous carbonaceous material. In contrast to the IDPs dominated by layer-lattice silicates, these particles generally show a great deal of void space.

In addition to their major minerals, both types of chondritic IDPs contain amorphous carbonaceous material and a wide variety of secondary minerals, several of which have never been seen in natural samples before. Some of the more common secondary minerals include magnetite, hematite, various iron-nickel sulfides and carbides, and carbonates. Particularly conspicuous by its absence is graphite. The carbonaceous materials are generally amorphous. Many of the particles also contain small regions of amorphous or 'glassy' material which have roughly solar elemental abundances.

An important point to note is that while a variety of crystalline minerals are often found together in the same particle, they are usually not in chemical and mineralogical equilibrium with each other. This means that the various minerals did not all form together but were instead formed in different environments and then brought together. Thus, the crystalline silicates we presently see in the IDPs were not formed by a heating event that annealed a previously amorphous silicate grain. This implies that the collected IDPs represent the end products of earlier aggregation processes. More detailed discussions of the morphology and mineralogy of IDPs can be found in Fraundorf (1981), Mackinnon and Rietmeijer (1987), and Bradley (1988).

At this point it is worth making several general observations about the morphology of the collected IDPs. First, neither whole IDPs nor their constituent subgrains show any appreciable tendency to be elongated. Only very exceptional IDPs or mineral grains have length-to-width ratios greater than 1.5. The collected particles do 


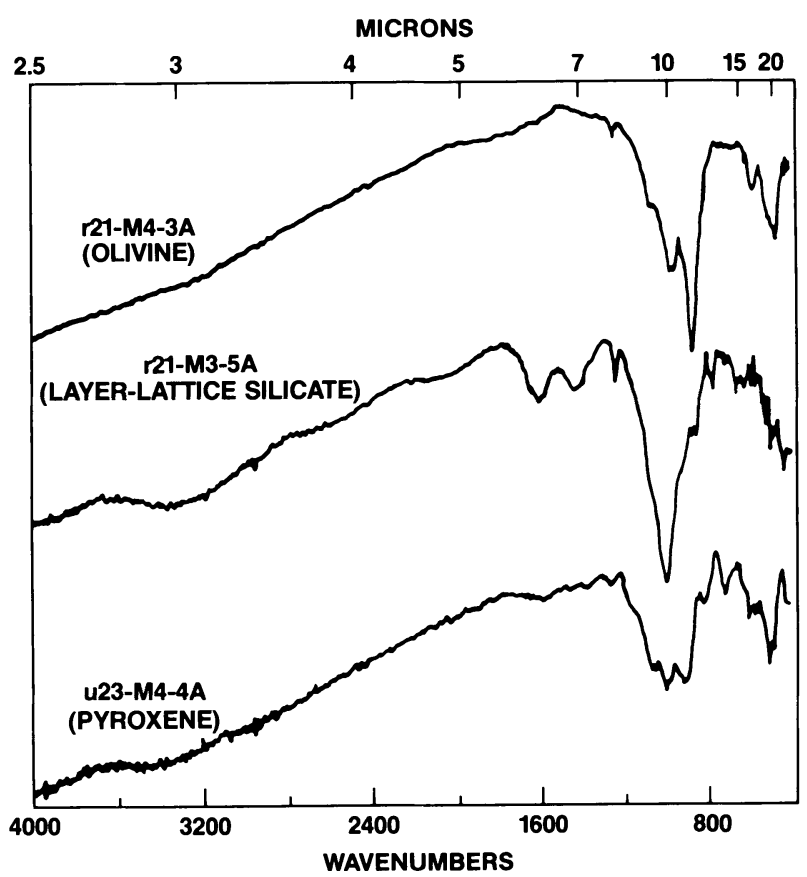

Fig. 3. Representative infrared spectra from the three main IDP infrared classes. From top to bottom the spectra are dominated by the characteristic absorption features of olivines, layer-lattice silicates, and pyroxenes, respectively. In addition to the " $10 \mu \mathrm{m}$ " and " $20 \mu \mathrm{m}$ " silicate features, the spectra dominated by layer-lattice silicates also show features near 3.0 and $6.0 \mu \mathrm{m} \mathrm{(3330} \mathrm{and} 1670$ $\left.\mathrm{cm}^{-1}\right)$ due to hydration and a feature near $6.9 \mu \mathrm{m}\left(1450 \mathrm{~cm}^{-1}\right)$ due to carbonates. (Figure adapted from Sandford and Walker, 1985).

not bear any strong resemblance to model grains produced by 'fractal' coagulation, although it must be remembered that the atmospheric entry process will selectively bias against more fragile structures. Finally, neither whole collected IDPs nor their components show much evidence for the presence of classical 'core-mantle' grains.

\section{IDP SPECTRAL PROPERTIES AND ASTRONOMICAL COMPARISONS}

The infrared spectral properties of the collected IDPs are of great interest since they provide the major means by which we can compare the collected dust to dust in the interplanetary medium and comets, and the only means by which we can compare the IDPs to dust in the interstellar medium. The infrared (2.5-25 $\mu \mathrm{m}, 4000-400$ $\mathrm{cm}^{-1}$ ) spectra of IDPs can be separated into groups based on the observed profile of their " $10 \mu \mathrm{m}$ " silicate features (Sandford and Walker, 1985). As mentioned earlier, these groups refer to particles dominated by olivines, pyroxenes, and layer-lattice 
silicates (Figure 3). Spectra from the olivine-rich particles show the characteristic " 10 " and " 20 " $\mu \mathrm{m} S i-O$ stretching and $S i-O-S i$ bending mode profiles of olivine. The same is true for the pyroxene-rich particles, although there is greater variation in the profiles, presumably because of the greater compositional variety available in pyroxenes. In addition to the appropriate characteristic silicate features, the spectra of particles dominated by layer-lattice silicates typically show several other bands as well. These additional features are near 3.0 and $6.0 \mu \mathrm{m}$ (3330 and 1670 $\mathrm{cm}^{-1}$ ) (due to the $O-H$ stretching and $H-O-H$ bending modes, respectively, of adsorbed and absorbed water) and near 6.9 and $11.4 \mu \mathrm{m}\left(1450\right.$ and $\left.880 \mathrm{~cm}^{-1}\right)$ (due, respectively, to the stretching and scissoring modes of carbonates).

While the infrared spectra of IDPs are dominated by the minerals in the particles, the interaction of the particles with visible light is dominated by the carbonaceous material in the grains (Allamandola, Sandford, and Wopenka, 1987; Wopenka, 1988). This is demonstrated by the very different vibrational spectra measured using the Raman effect (Figure 4). Several interesting points are revealed by the Raman spectra. First, the majority of the spectra are dominated by first-order (1350 and $1600 \Delta \mathrm{cm}^{-1}$ ) and second-order (between 2200 and $3300 \Delta \mathrm{cm}^{-1}$ ) features characteristic of disordered carbonaceous materials rich in aromatic molecular units. Comparison of the relative band strengths and widths with laboratory standards suggests that the aromatic moieties responsible are smaller than $25 \AA$ in size. Second, some of the spectra show luminescence in the red portion of the spectrum. Finally, while the particles are dominated in mass by silicates, silicate features are only rarely found in the Raman spectra. This suggests that most of the silicates are effectively 'embedded' in carbonaceous material.

It is instructive to compare the spectra of the IDPs with the spectra of various astronomical objects. Caution is warranted, however, as the IDPs are comprised of refractory materials, whereas many of the astronomical sources contain volatiles as well. For example, many of the collected IDPs are likely to be derived from (volatilerich) comets, objects which have been suggested to be reservoirs of the primordial interstellar dust from which our solar system formed. Figure 5 shows a $5-13 \mu \mathrm{m}$ spectrum of Comet Halley taken on 12.1 December 1985 (Bregman et al., 1987). The solid line is a fit to the data using a composite IDP spectrum. The composite spectrum consists of a mixture of roughly $65 \%$ olivine-rich, $35 \%$ pyroxene-rich, and $10 \%$ layer-lattice silicate-rich IDPs. Not only is the "10 $\mu \mathrm{m}$ " silicate feature reasonably well-fitted, but the Halley spectrum also shows a feature near $6.9 \mu \mathrm{m}$ $\left(1450 \mathrm{~cm}^{-1}\right)$ which is consistent with the presence of carbonates in the abundances in which they are normally found in the IDPs rich in layer-lattice silicates. Similar fits with the IDP data have also been made to spectra from Comets Wilson and Giacobinni-Zinner. The ability of the IDP spectra to fit the cometary spectra and the detailed structure within the cometary silicate feature tells us undeniably that comets contain crystalline silicates.

It is also interesting to compare spectroscopic properties of the carbonaceous component in IDPs with the spectra of various astronomical objects. Figure 6 shows a comparison between the 5-10 $\mu \mathrm{m}\left(1000-2000 \mathrm{~cm}^{-1}\right)$ emission from the Orion bar and the 1000-2000 $\Delta \mathrm{cm}^{-1}$ Raman spectrum of an IDP. Anyone familiar with the recent suggestions that the interstellar infrared emission bands are due to polycyclic aromatic hydrocarbons (PAHs) or PAH-related materials will recognize this figure with the exception that the interstellar emission is not compared to the Raman spectrum of auto soot but instead to the spectrum of actual extraterrestrial material. 


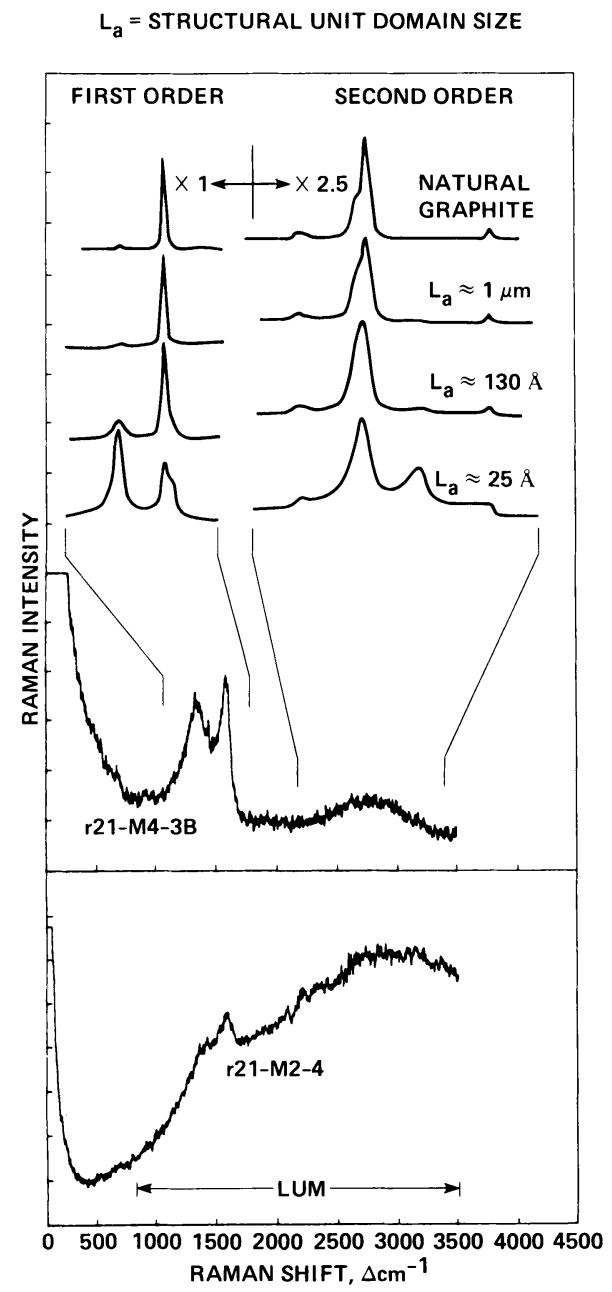

Fig. 4. The Raman spectra of two IDPs (bottom). Superposed above the IDP spectra are the Raman spectra of carbonaceous laboratory standards of increasing disorder. The dominance of features near 1350, 1600, and $2700 \Delta \mathrm{cm}^{-1}$ indicate that aromatic $C-C$ modes are responsible. Comparison between the IDP and standard spectra indicate that the aromatic units in IDPs are generally smaller than $25 \AA$ in size. The IDP samples were excited by $5145 \AA$ photons, the lab standards by $4880 \AA$ photons. The lab standards spectra are taken from Nemanich and Solin (1979). The IDP spectra are from Allamandola, Sandford, and Wopenka (1987). 


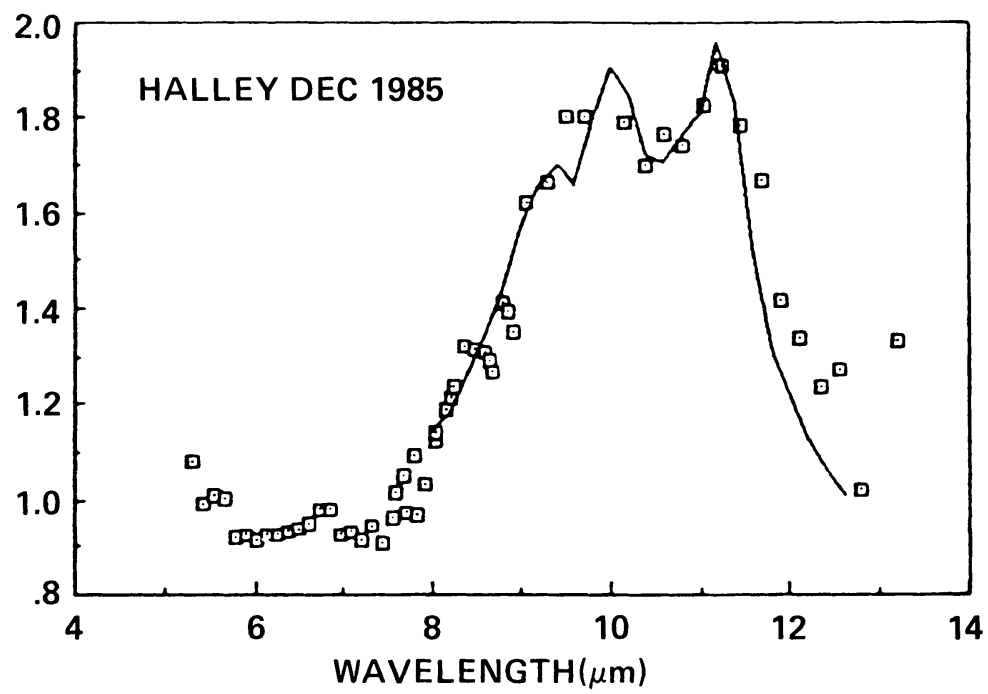

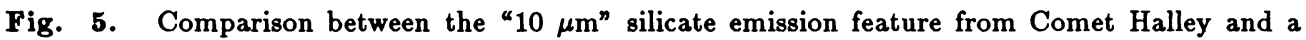
composite of IDP spectra. The IDP composite spectrum contains IDP contributions in the relative proportion of $55 \%$ olivine, $35 \%$ pyroxene, and $10 \%$ layer-lattice silicates and has been adjusted to correspond to dust at $320 \mathrm{~K}$. (Figure adapted from Bregman et al., 1987).

The similarity in all the spectra suggest that aromatic molecular units of some kind are responsible. One should be careful, however, not to over-interpret the match in Figure 6 (or in the analogous Orion-soot match). For a vibrational mode to be infrared active, it must involve a change in the dipole moment of the molecule, while to be Raman active it must involve a change in the polarizability of the molecule. Thus, there is no a priori reason to expect the infrared emission (or absorption) spectrum of a material to identically match its Raman spectrum. The favorable comparison in Figure 6 demonstrates that the two spectra are produced by a mixture of materials having vibrations that fall in the same frequency range, and thus presumably materials having similar (i.e. aromatic) chemical bonds.

\section{ISOTOPIC EVIDENCE OF AN INTERSTELLAR COMPONENT}

Unfortunately, our ability to fit the spectra of IDPs to cometary and interstellar spectra in no way proves that the collected particles contain an interstellar component. What evidence is there, then, that the collected IDPs contain interstellar material? The major evidence that the collected IDPs contain some interstellar material comes from isotopic studies.

Many of the collected IDPs are found to contain large deuterium enrichments $\left(D / H>2 \times 10^{-4}\right.$ and as high as at least $\left.1.5 \times 10^{-3}\right)$ which are suggestive of an interstellar origin (McKeegan, Walker, and Zinner, 1985). The deuterium enrichments seem to correlate loosely with carbon concentration, suggesting a carbonaceous carrier. Measurable deviations in the ${ }^{13} C /{ }^{12} C$ ratio are found in IDPs, but these effects 

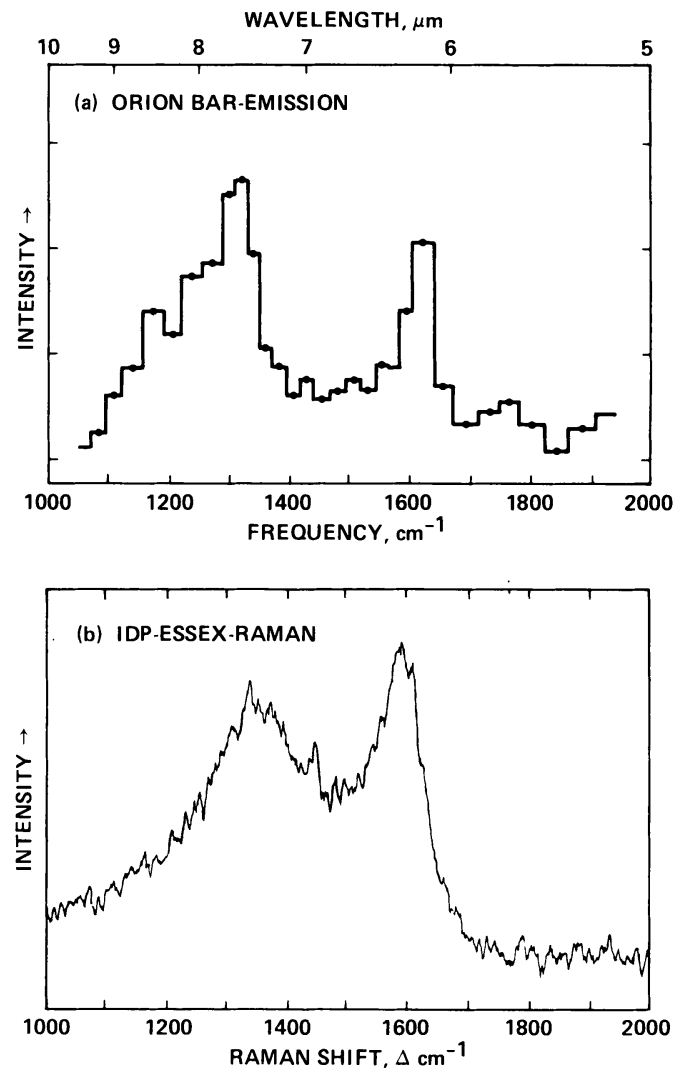

Fig. 6. Comparison between the " $6.2^{\text {" }}$ and " $7.7 \mu \mathrm{m}$ " interstellar emission features and the Raman spectrum of an IDP. The similarity in the spectra suggests aromatic compounds are responsible for both sets of features. The Orion spectrum is taken from Bregman et al. (1984) and the IDP spectrum is from Allamandola, Sandford, and Wopenka (1987).

are small and are uncorrelated with the deuterium anomalies. The spatial distribution of the carrier of the deuterium excess is not uniform, but instead, in at least some particles, seems to be confined to 'hot spots' whose dimensions are smaller than a micron. Unfortunately, due to the small size of the carrier, it is not yet clear whether the deuterium-rich 'hot spots' represent true, unaltered interstellar grains that have been incorporated unmodified into the larger IDP, or whether they represent material partially-altered in the solar nebula which still has a 'molecular memory' of such a grain.

The origin of the deuterium excess is somewhat uncertain. Deuterium enrichment via chemical reactions requires very low temperatures $(T<100 \mathrm{~K})$, but at these temperatures the reaction rate is extremely slow. The reaction threshold is greatly reduced, however, if one of the reactants is an ion. For this reason, it is felt that ion-molecule reactions taking place in dense, cold interstellar clouds may 
be the source of the observed deuterium enrichments (cf. Yang and Epstein, 1983; and references therein). Molecules formed under these conditions were presumably incorporated into interstellar dust grains, some of which subsequently survived in the solar nebula.

An additional means by which deuterium enrichment can occur in the interstellar medium involves selective photodissociation reactions (Allamandola, Sandford, and Wopenka, 1987). It has been suggested that free molecular PAHs are responsible for the infrared emission bands seen in many emission nebulae (Léger and Puget, 1984; Allamandola, Tielens, and Barker, 1985). Interactions of ultraviolet photons with these molecules will occasionally result in the photodissociation of one of the molecules' peripheral hydrogen atoms. Since the zero point energy of the $C-D$ bond in PAHs is lower than that of the $C-H$ bond, deuterium is less likely to be dissociated. Once a hydrogen is removed, that bond site is available for the capture of a new atom. Usually another hydrogen atom will link to the dangling bond, but occasionally a deuterium may be captured. If this process is allowed to continue, it will eventually result in deuterium enrichment of the molecular PAH population. In any event, whether the observed enrichments are produced via ionmolecule reactions, selective photodissociation, or an as yet unrecognized process, their presence in IDPs clearly suggests material having an interstellar origin.

It should be noted, however, that while the presence of an exotic isotopic component suggests that interstellar material is present, the lack of an exotic isotopic signature does not necessarily imply formation in the solar system. Since the solar system is presumably derived from more-or-less normal interstellar material, we might expect most interstellar material to have 'normal', i.e. solar system, isotopic abundances. Thus, the interstellar material evidenced by the deuterium enrichments represents, in some sense, a lower limit to the abundance of interstellar material present.

\section{IMPLICATIONS}

Given that the collected IDPs may contain interstellar material, it is interesting to consider three alternative scenarios.

Possibility 1: Individual collected IDPs represent actual, unaltered interstellar grains. In this case, the grains presumably formed in the interstellar medium by aggregation of previously unrelated grains formed in a variety of environments. If this is the case, then models of the interstellar medium should include the physical and spectral behavior of composite grains consisting of crystalline silicates and amorphous carbonaceous material that are mixed more-or-less randomly. The interaction of visible light with the particles would be controlled largely by the carbonaceous component, even when the silicates are more abundant than the carbonaceous materials by mass by factors as high as 40 .

Possibility 2: All of the compositional subunits found in the collected IDPs have interstellar origins, but they did not form an aggregate particle until they entered the solar nebula. This possibility results in the same consequences as the previous case with the relaxed constraint that composite aggregates need not necessarily be considered for interstellar grains. 
Possibility 3: The majority of the material in the collected IDPs consists of interstellar material that has been sufficiently reprocessed in the solar nebula to lose most or all evidence of its original form, but the particles contain some interstellar components carrying isotopic anomalies which survived incorporation and subsequent storage in the solar nebula. This case is probably the closest to reality and, unfortunately, provides the fewest constraints. Since the exact mineralogical and elemental nature of the carriers of the isotopic anomalies are presently not known, they provide few clues.

\section{SUMMARY}

The information obtained from IDPs suggests several important points be considered:

(i) IDPs show little or no evidence for the presence of well-ordered graphite. The carbonaceous material present is amorphous.

(ii) Nearly all IDPs are mineralogically heterogeneous, implying that they formed by coagulation of smaller precursors. Composite grains may be very common in interstellar space.

(iii) The Raman and luminescence spectra of IDPs suggest that carbonaceous 'coatings' are likely to surround many silicate grains. The thickness of these 'coatings' is uncertain in IDPs, but it is sufficient to screen the silicates from most visible photons (i.e. greater than about $100 \AA$ ). There is no evidence, however, that the 'coatings' have 'classic' core-mantle morphologies.

(iv) Most of the silicates in IDPs are crystalline, not amorphous, and the possibility that interstellar grains may contain some crystalline silicates should be seriously considered. Amorphous silicates are metastable and become more ordered if heated. Even if interstellar silicates are formed as amorphous materials, some will become more crystalline during heating in mild shocks, grain-grain collisions, etc. Thus, the presence of amorphous silicates will unavoidably lead to the presence of some crystalline silicates. Evidence for this crystalline component in interstellar dust might best be found by searching for weak spectral structure in the "10 $\mu \mathrm{m}$ " silicate band.

(v) There is no evidence from IDPs that natural dust populations are likely to contain large amounts of elongated grains. Irregular, roughly equidimensional grains are the most common.

ACKNOWLEDGEMENTS. The author would like to thank Drs. Robert M. Walker and Louis J. Allamandola for critical readings of previous versions of this paper.

\section{REFERENCES}

Allamandola, L. J., Tielens, A. G. G. M., and Barker, J. R. 1985, Ap. J. (Letters), 290, L25-L28. Allamandola, L. J., Sandford, S. A., and Wopenka, B. 1987, Science, 237, 56-59.

Bradley, J. P. 1988, Geochim. Cosmochim. Acta., 52, 889-900. 
Bradley, J. P., Sandford, S. A., and Walker, R. M. 1988, in Meteorites and the Early Solar System, eds. J. Kerridge and M. Matthews, (Tucson: Univ. Ariz. Press), in press.

Bregman, J., Allamandola, L. J., Simpson, J., Tielens, A. G. G. M., and Witteborn, F. 1984, in Airborne Astronomy, NASA CP-2353, p. 140-147.

Bregman, J. D., Campins, H., Witteborn, F. C., Wooden, D. H., Rank, D. M., Allamandola, L. J., Cohen, M., and Tielens, A. G. G. M. 1987, Astr. Ap., 187, 616-620.

Fraundorf, P. 1981, Geochim. Cosmochim. Acta., 45, 915-943.

Fraundorf, P., Brownlee, D. E., and Walker, R. M. 1982, in Comets, ed. L. L. Wilkening, (Tucson: Univ. Ariz. Press), p. 383-409.

Léger, A., and Puget, J. L. 1984, Astr. Ap., 137, L5-L8.

Mackinnon, I. D. R., and Rietmeijer, F. J. M. 1987, Rev. Geophys., 25, 1527-1553.

McKeegan, K. D., Walker, R. M., and Zinner, E. 1985, Geochim. Cosmochim. Acta., 49, 19711987.

Nemanich, R. J., and Solin, S. A. 1979, Phys. Rev. B, 20, 392-401.

Sandford, S. A. 1987, Fund. Cosmic Phys., 12, 1-73.

Sandford, S. A., and Walker, R. M. 1985, Ap. J., 291, 838-851.

Wopenka, B. 1988, Earth Planet. Sci. Letters, 88, 221-231.

Yang, J., and Epstein, S. 1983, Geochim. Cosmochim. Acta., 47, 2199-2215. 


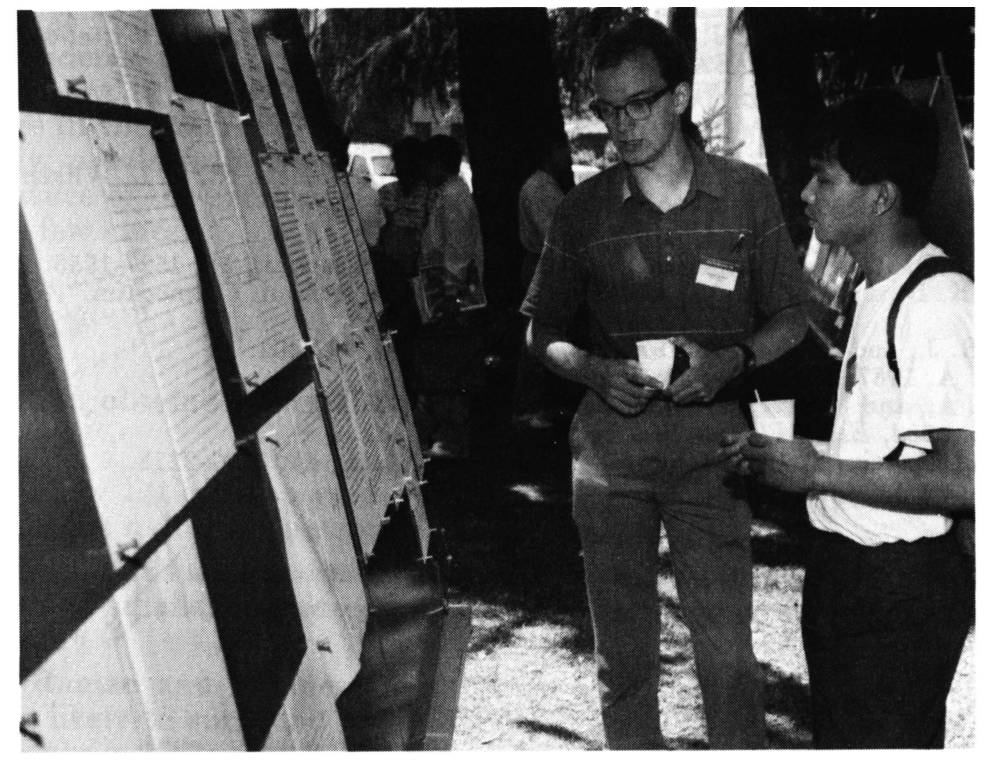

Harold Butner and Rene Laurijs 